\title{
Responses of Human Motoneurons to Corticospinal Stimulation during Maximal Voluntary Contractions and Ischemia
}

\author{
Jane E. Butler, Janet L. Taylor, and Simon C. Gandevia \\ Prince of Wales Medical Research Institute and University of New South Wales, Randwick, New South Wales, 2031 Australia
}

\begin{abstract}
The discharge frequency of human motoneurons declines during a sustained isometric maximal voluntary contraction (MVC) of elbow flexor muscles, but the cause is unresolved. We aimed to determine whether motoneurons were inhibited during a sustained fatiguing contraction of the elbow flexor muscles and whether this inhibition was caused by the discharge of group III and IV muscle afferents. Subjects performed brief MVCs before and after a fatiguing $2 \mathrm{~min}$ MVC. During maximal efforts, electromyographic responses recorded from the elbow flexor muscles were evoked by stimulation of the corticospinal tracts at the cervicomedullary level [cervicomedullary motor evoked potentials (CMEPs)] and by supramaximal stimulation over the brachial plexus $\left(M_{\max }\right)$. This revealed a novel decrease in the size of the muscle response to corticospinal tract stimulation during fatigue. During the sustained MVCs, the size of CMEPs decreased to $81 \pm 15$ and $78 \pm 15 \%$ of the control value for brachioradialis and biceps brachii, respectively (mean \pm SEM; $n=8$ ). This recovered within $15 \mathrm{sec}$ after the fatiguing contraction. In a second set of studies, input from group III and IV muscle afferents was prolonged after the end of the fatiguing contraction by holding the muscle ischemic with a cuff inflated above arterial pressure. Despite the maintained discharge of group III and IV afferents, the CMEPs again recovered within $15 \mathrm{sec}$ of the end of the sustained contraction. These results show a diminished output of spinal motoneurons to stimulation of corticospinal tracts during a fatiguing MVC; however, the mechanisms responsible for this decline are not attributable to activity in group III and IV muscle afferents.
\end{abstract}

Key words: fatigue; motoneuron (motor neuron); group III and IV muscle afferents; human; corticospinal; ischemia

\section{Introduction}

During a sustained isometric maximal voluntary contraction (MVC) the discharge rate of motoneurons usually declines. This decline has been observed for intrinsic muscles of the hand (Marsden et al., 1969, 1983; Bigland-Ritchie et al., 1983a,b; Gandevia et al., 1990), biceps brachii (Bigland-Ritchie et al., 1986; Woods et al., 1987), and tibialis anterior (Grimby et al., 1981; Macefield et al., 2000). Several mechanisms have been proposed to account for the reduction in firing rate. The most popular explanation is that activity in group III and IV muscle afferents reflexively inhibits spinal motoneurons. The strongest argument in favor of a causative role for group III and IV muscle afferents is that the firing rates of motoneurons (and voluntary force) remain depressed as long as the muscles are held ischemic after the contraction ceases (Bigland-Ritchie et al., 1986, 1995; Woods et al., 1987; Garland and Gossen, 2002). With the muscle at rest, motoneuronal and supraspinal factors should recover, whereas firing of group III and IV muscle afferents, which are sensitive to ischemia and metabolites, will be maintained while blood flow is occluded (Kaufman et al., 1984; Hayward et al., 1991; Taylor et al., 2000c). Further evidence for this view comes from the pres-

Received July 8, 2003; revised Sept. 14, 2003; accepted Sept. 16, 2003.

This work was funded by the National Health and Medical Research Council of Australia (\#3206).

Correspondence should be addressed to Dr. S. C. Gandevia, Prince of Wales Medical Research Institute, Barker Street, Randwick, New South Wales, Australia 2031. E-mail: s.gandevia@unsw.edu.au.

Copyright $\odot 2003$ Society for Neuroscience $\quad$ 0270-6474/03/2310224-07\$15.00/0 ence of a contraction-induced spinal inhibition between synergists in the cat hindlimb (Hayward et al., 1988; Sacco et al., 1997) as well as studies combining electrically induced contractions, H-reflexes, and partial nerve blocks (Garland and McComas, 1990; Garland, 1991; Walton et al., 2002). However, the reflex effects of group III and IV muscle afferents on spinal motoneurons include both excitatory and inhibitory inputs (Kniffki et al., 1979, 1981a,b), and the net effect on spinal motoneuronal excitability is unclear.

Other mechanisms that could contribute to the fatigueinduced decline in motoneuron firing rate include intrinsic motoneuronal properties (Kernell and Monster, 1982a,b; Spielmann et al., 1993; Sawczuk et al., 1997), changes in net input from other muscle receptors [e.g., muscle spindles (Bongiovanni and Hagbarth, 1990; Gandevia et al., 1990; Macefield et al., 1991, 1993)], and a decline in effective descending drive to the motoneurons (Gandevia et al., 1996; Taylor et al., 2000a).

The present studies were designed to test motoneuronal excitability during sustained maximal contractions by stimulation of descending motor paths at the cervicomedullary level. This method can activate the same corticospinal axons as transcranial magnetic stimulation of the motor cortex as judged by collision experiments (Ugawa et al., 1994; Gandevia et al., 1999; Taylor et al., 2002). It causes a monosynaptic EPSP in motoneurons of elbow flexors (Petersen et al., 2002), and the evoked responses are not influenced by conventional presynaptic inhibition (Nielsen 
A

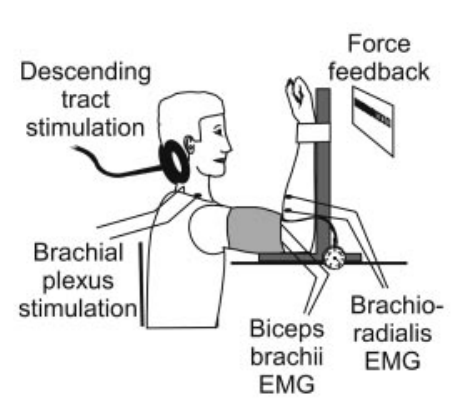

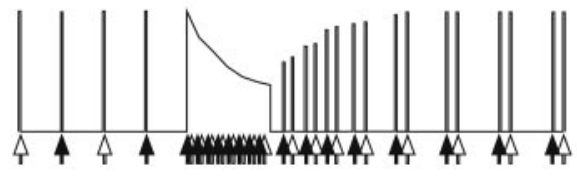

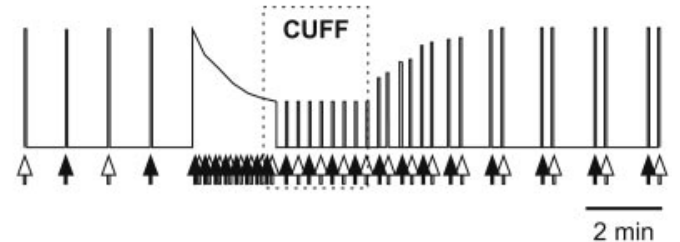

Figure 1. Experimental setup and protocol. $A$, Experimental setup. $B$, Two protocols. Brief control MVCs were performed during which stimuli were delivered to the brachial plexus (white arrows) or to the corticospinal tracts (black arrows). The subject then performed a 2 min sustained MVC during which stimuli were delivered alternately to the corticospinal tracts and the brachial plexus. Brief recovery contractions were performed afterward. In the second protocol (bottom panel), the forearm muscles were held ischemic for 2 min after the sustained MVC (region labeled CUFF).

and Petersen, 1994). Maintained muscle ischemia was used to prolong the firing of group III and IV afferents after the end of the sustained MVC (Kaufman et al., 1984; Taylor et al., 2000c) and thus to reinvestigate the proposition that these afferents produce significant inhibition of motoneurons during fatiguing voluntary contractions.

Preliminary results have been published previously (Butler et al., 1999).

\section{Materials and Methods}

The main experiments were performed on eight subjects (three females) who were studied on two occasions. Additional control experiments (described below) were also performed on eight subjects (four females) on two occasions. Three of the subjects performed both the main and control experiments. Subjects were healthy and ranged in age from 30 to 58 years. The protocols were approved by the local ethics committee, and the studies were conducted according to the Declaration of Helsinki. All subjects gave informed written consent.

\section{Experimental task and recordings}

Many of our experimental procedures and recording techniques are similar to those described previously (Gandevia et al., 1999; Taylor et al., 2000c). The test fatiguing contraction was a sustained maximal voluntary effort (MVC) of the right elbow flexors lasting $120 \mathrm{sec}$. The elbow was flexed to $90^{\circ}$ and strapped to an isometric myograph (Fig. 1 A) (Allen et al., 1995). Before the test contraction a series of brief MVCs (lasting 2-3 $\mathrm{sec}$ ) were performed at $\sim 1 \mathrm{~min}$ intervals. After the test contraction, brief MVCs were performed (at 15, 30, 45, 60, 75, 90, 120, 135, 180, 195, 240, 255,300 , and $315 \mathrm{sec}$ after the sustained contraction) to assess the recovery of force and the electromyographic (EMG) responses to stimulation (Fig. $1 B$ ). Subjects received visual feedback of force and constant verbal encouragement to contract maximally throughout every contraction. This protocol was repeated several days later with one change: a sphygmomanometer cuff wrapped around the upper arm was inflated to 300 $\mathrm{mmHg}$ from a source of compressed air with $15 \mathrm{sec}$ remaining in the test contraction. Inflation of the cuff was maintained for $120 \mathrm{sec}$ into the recovery period during which eight additional brief recovery MVCs were performed every $15 \mathrm{sec}$. After the cuff was deflated the subjects performed more brief recovery MVCs (at 15, 30, 45, 60, 75, 90, 120, 135, 180, $195,240,255,300,315,360$, and $375 \mathrm{sec}$ after cuff deflation).

Surface electrodes over brachioradialis and biceps brachii recorded $\mathrm{EMG}$ responses ( $\mathrm{Ag}-\mathrm{AgCl}$ electrodes, $10 \mathrm{~mm}$ diameter). The signals were amplified (100-300×), filtered (1.6 Hz-1 kHz; CED 1902 amplifiers), and recorded via a CED 1401 interface with Signal software (sampling rate $5 \mathrm{kHz}$ ) (Cambridge Electronic Design, Cambridge, UK).

\section{Stimulation}

Brachial plexus stimulation. Stimuli were delivered to the brachial plexus at Erb's point so that the maximal $M$ wave could be monitored $\left(M_{\max }\right)$. The stimulus intensity was at least $50 \%$ above that required to produce a maximal response in both brachioradialis and biceps brachii. The cathode was in the supraclavicular fossa, and the anode was over the acromion. Stimuli were rectangular pulses $(100 \mu \mathrm{sec}$ duration) delivered via a Digitimer DS7 constantcurrent stimulator (modified for outputs up to 1 A; Digitimer Ltd., Welwyn Garden City, UK).

Corticospinal tract stimulation. Stimuli were also delivered to corticospinal tracts close to the cervicomedullary junction. Responses evoked by cervicomedullary stimulation are termed cervicomedullary motor evoked potentials (CMEPs). For two of the eight subjects, corticospinal stimulation was achieved using an electrical stimulus passed between two $9 \mathrm{~mm} \mathrm{Ag}-$ $\mathrm{AgCl}$ electrodes filled with conductive paste and glued to the skin over the left (cathode) and right (anode) mastoid processes (Ugawa et al. 1991; Gandevia et al., 1999). The pulse was $100 \mu$ sec duration, and the intensity range was 360-540 V (Digitimer, D180). For six subjects, CMEPS were evoked using a Magstim 200 with a double-cone coil from which the plastic casing had been removed (Magstim Company Ltd., Dyffed, UK). This allowed the center of the coil to be closely opposed to the back of the skull. Usually the coil center was positioned $\sim 2 \mathrm{~cm}$ below the inion, but sometimes it was moved to the right by $\sim 1-2 \mathrm{~cm}$ to produce adequate responses. Current direction was downward in the coil (Ugawa et al., 1994; Taylor et al., 2000b). Stimulus intensities were between 80 and $100 \%$ stimulator output to evoke responses during brief control MVCs of $\sim 60-70 \% M_{\max }$. At this intensity the latency of the responses was $8.4 \pm 0.6 \mathrm{msec}$ (mean $\pm \mathrm{SD}$ ) for biceps and $11.2 \pm 1.1$ $\mathrm{msec}$ for brachioradialis. Responses were monitored at high gain to ensure that the onset of the CMEPs was not contaminated by activation of axons distal to the cell body of the motoneurons.

For the main experiment, during the brief control and recovery MVCs, stimuli to either the brachial plexus or the corticospinal tracts were delivered alternately at the peak force of each MVC. During the sustained MVC, corticospinal tract and brachial plexus stimuli were delivered in pairs ( $5 \mathrm{sec}$ apart) every $15 \mathrm{sec}$ throughout the $2 \mathrm{~min}$ contraction.

\section{Additional control experiments: submaximal brachial plexus stimulation}

Because the size of the compound muscle action potential can change with exercise (Cupido et al., 1996; Taylor et al., 1999), the size of the CMEPs was always normalized to the compound muscle action potential ( $M$ wave) produced at a nearby time by supramaximal stimulation of the brachial plexus (i.e., CMEPs were expressed as a percentage of $M_{\max }$ ). This procedure has been used previously (Gandevia et al., 1999; Taylor et al., 2000c). A concern with this normalization was that the sustained muscle contraction and the maintained ischemia for 2 min may have different effects on the sarcolemmal function of different-sized muscle fibers such that the change in size of $M_{\max }$ may not reflect the changes in the muscle fibers recruited in a submaximal CMEP (Enoka et al., 1992). If this occurred, it would not be appropriate to normalize a submaximal muscle action potential to $M_{\max }$. Therefore, additional control experiments were performed in eight subjects using both supramaximal and submaximal stimulation of the brachial plexus to test whether a sustained MVC with maintained muscle ischemia produced differential effects on the maximal muscle action potential $\left(100 \% M_{\max }\right)$ compared with the submaximal muscle action potential $\left(\sim 65 \% M_{\max }\right.$, a level that would be comprised of larger rather than smaller motor units). The experiment focused on responses in brachioradialis because this muscle is distal to the sphygmomanometer cuff. The two protocols of the main experiment described above were repeated except that the corticospinal stimulus was 


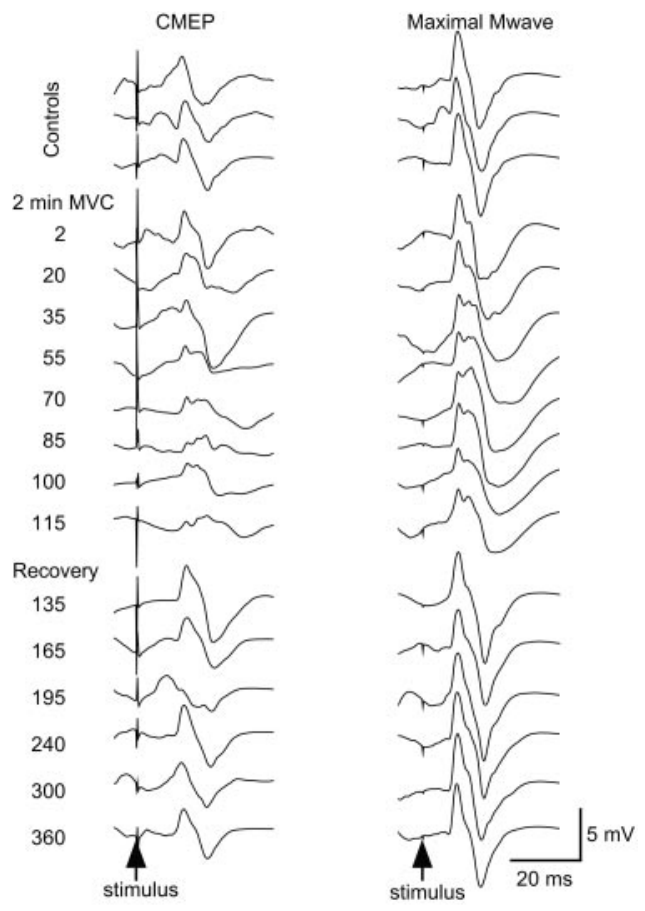

Figure 2. Single trials of EMG from a typical subject without maintained muscle ischemia. Single responses from brachioradialis to stimulation of the corticospinal tracts (CMEP, left panels) and the brachial plexus ( $M_{\max }$, right panels) from a typical subject without a period of postcontraction muscle ischemia. All responses were obtained during maximal efforts: responses during brief control MVCs (top 3 traces), the 2 min sustained MVC (middle 8 traces), and brief recovery MVCs (bottom 6 traces). The time of corticospinal tract stimulation is indicated on the left with brachial plexus stimulation 5 sec later.

replaced by the submaximal stimulus to the brachial plexus. In one protocol the subjects made a sustained MVC for $120 \mathrm{sec}$ followed by brief MVCs in the recovery period every $15 \mathrm{sec}$. In the second, an identical protocol was performed with the only differences being that the cuff was inflated with $15 \mathrm{sec}$ remaining in the test contraction and was maintained for $120 \mathrm{sec}$ into the recovery period, as in the main experiment above. After the cuff was deflated the subjects performed more brief recovery MVCs every $15 \mathrm{sec}$. The size of the submaximal and maximal $M$ waves were compared during 2 min MVCs with and without subsequent ischemia. Submaximal $M$ wave responses were normalized to the nearest maximal $M$ wave for each experiment. The normalized submaximal responses of the two additional control experiments were compared.

\section{Analysis}

Throughout the text, the area of the CMEPs is reported, although similar results occurred if the peak-to-peak amplitude was used. The area of the CMEPs was measured between cursors appropriately positioned for each potential depending on its duration. Because the conduction velocity of the muscle fiber action potential slows with fatigue, the duration of the responses increases. During the $2 \mathrm{~min}$ MVC, each CMEP was normalized to $M_{\max }$ elicited within $5 \mathrm{sec}$ of the CMEP, whereas during recovery CMEPs were normalized to $M_{\max }$ elicited during the next brief MVC (15 sec later). A two-way ANOVA was performed (with Student-NewmanKeuls post hoc tests) to test differences between four time periods (the three control responses, the three responses in the first $40 \mathrm{sec}$ of the MVC, the three responses in the last $40 \mathrm{sec}$ of the MVC, and the three responses in the first $90 \mathrm{sec}$ of recovery) for brachioradialis and biceps brachii during the experiments with and without muscle ischemia. Statistical significance was set at $p<0.05$.

\section{Results}

\section{Corticospinal tract stimulation}

During the 2 min sustained MVC, there were changes in the size of the responses in brachioradialis and biceps brachii produced both by corticospinal stimulation and brachial plexus stimulation (Figs. 2, 4). Both the CMEP and the $M_{\max }$ initially increased in area compared with responses evoked during the initial brief control MVCs. In the latter half of the sustained contraction the size of both responses fell. The CMEP decreased to control values, whereas $M_{\max }$ remained larger than its control size (Fig. $3 A$ ). Thus, relative to $M_{\max }$, the CMEP decreased during the fatiguing contraction. Both the CMEP and $M_{\max }$ returned close to control levels within $15 \mathrm{sec}$ after the sustained MVC.

Because changes in $M_{\max }$ must also influence the CMEP, all responses to cervicomedullary stimulation were normalized to $M_{\max }$ elicited under similar conditions (see Materials and Methods). For the 2 min MVC they were normalized to $M_{\max }$ responses elicited within $5 \mathrm{sec}$, and for the brief recovery MVCs they were normalized to responses in the next MVC (15 sec later). Changes in the normalized CMEP should represent alterations in the response of the motoneurons.

The normalized CMEP decreased in area during the $2 \mathrm{~min}$ MVC and quickly recovered to control levels. In the first experiment, without maintained ischemia, the area of the CMEP in brachioradialis was initially $50 \pm 3 \% M_{\max }$ during the brief MVCs. It decreased to $39 \pm 5 \% M_{\max }$ late in the sustained contraction. The CMEP in biceps brachii also decreased by $\sim 20 \%$ from $65 \pm 4 \% M_{\max }$ to $50 \pm 6 \% M_{\max }$ (Fig. $3 B$ ). For each muscle, an ANOVA compared the control responses, the responses in the first $40 \mathrm{sec}$ of the MVC, the last $40 \mathrm{sec}$ of the MVC, and the first 90 sec of recovery. In both brachioradialis and biceps brachii the area of responses differed between these four time periods (brachioradialis, $p<0.001$; biceps brachii, $p<0.001$ ), and post hoc comparison of the means showed that responses near the end of the sustained MVC were decreased in each muscle compared with the control and recovery periods (Student-Newman-Keuls; $p<0.05$ ). Responses during the brief recovery contractions were not significantly different from control values.

To test whether group III and IV muscle afferents were responsible for the decrease in size of the normalized CMEP during the $2 \mathrm{~min}$ MVC, the experiment was repeated in the same subjects but with the muscles held ischemic for an additional 2 min after the end of the sustained contraction (Fig. 5). If group III and IV muscle afferents were responsible for the decrease in the CMEP, we would expect this decrease to be maintained throughout ischemia. As in the experiment in which the muscles were not held ischemic, the CMEP decreased relative to $M_{\max }$ during the $2 \mathrm{~min}$ MVC. In brachioradialis, the CMEP decreased from an initial $60 \pm 4 \%$ to $42 \pm 4 \% M_{\max }$ and in biceps brachii from $73 \pm 4 \%$ to $53 \pm 5 \% M_{\max }$. Again the CMEPs were significantly reduced in each muscle near the end of the sustained MVC, and the CMEPs evoked in the first $90 \mathrm{sec}$ of recovery were not different from control levels. This suggests that the maintained ischemia did not affect the recovery of CMEPs recorded during brief MVCs after the sustained contraction. Furthermore, when the responses evoked during the maintained ischemia at 15, 45, and $75 \mathrm{sec}$ after the end of the 2 min MVC were compared directly with those evoked at the same times but without maintained ischemia, no differences were seen in either muscle (paired $t$ tests, $p=0.31$, $p=0.65, p=0.64$ for brachioradialis, and $p=0.99, p=0.88, p=$ 0.24 for biceps brachii).

\section{Control experiments: submaximal brachial plexus stimulation}

For both experiments, with and without maintained muscle ischemia, the ratio of the submaximal $M$ waves to the maximal $M$ waves remained similar (Fig. 6). We found no changes in ratios of 
A
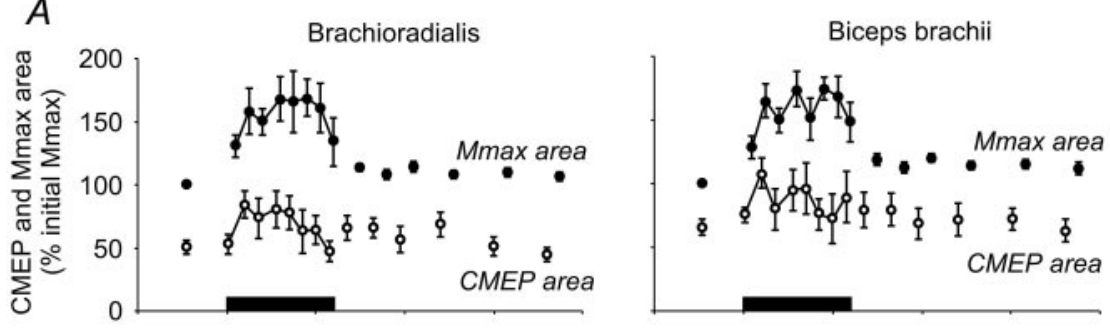

$B$
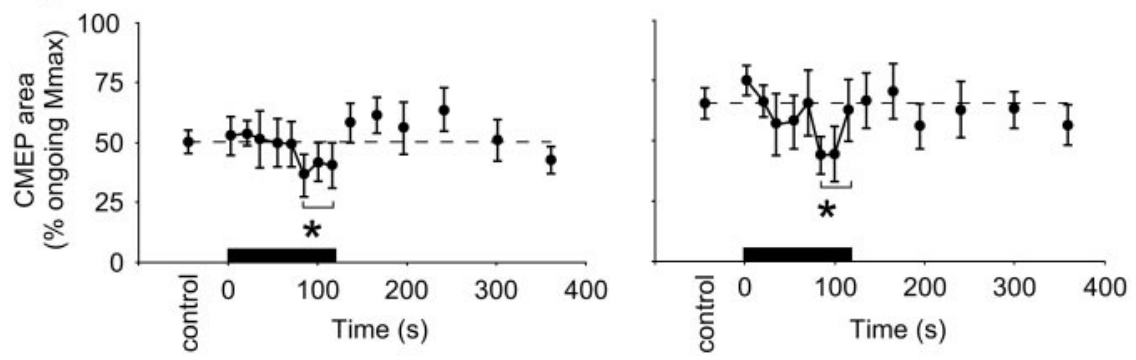

Figure 3. Pooled data for CMEP and $M_{\max }$ without maintained muscle ischemia. Data from brachioradialis (left panels) and biceps brachii (right panels) before, during, and after a 2 min sustained MVC ( $0-120$ sec; $n=8$ subjects). In each panel the solid bar indicates the timing of the sustained maximal effort. $A$, Panels show the mean area ( \pm SEM) of the responses to stimulation of the corticospinal tracts (CMEP, open circles) and the brachial plexus ( $M_{\max }$, filled circles) expressed as a proportion of the mean initial maximal $M$ wave (\%initial $M_{\max }$ ). Notice the increase in $M_{\max }$ during the sustained contraction. $B$, Panels show the mean CMEP area normalized to the $M_{\max }$ recorded at close to the same time (\% ongoing $M_{\max }$ ). During the 2 min sustained MVC the CMEP decreases and then recovers by the first recovery contraction to control size. The broken horizontal lines indicate the mean area of CMEPs during brief control contractions. ${ }^{*}$ CMEPs grouped over the last $40 \mathrm{sec}$ of sustained MVC were reduced compared with those in the control period, in the first 40 sec of sustained MVC and in the first 90 sec of recovery $(p<0.05)$. the submaximal to maximal responses (i.e., responses in the larger motor units vs all motor units) either during the sustained MVC or during subsequent ischemia for biceps brachii or brachioradialis. This indicates that for brachioradialis, there was no differential effect or change in the sarcolemma affecting different motor unit types as a result of the muscle ischemia. Thus, we concluded that expression of the size of the CMEP relative to $M_{\max }$ was appropriate to reveal the effects of motoneuronal changes on the CMEP and to exclude changes at the level of the muscle fiber.

\section{Discussion}

This study has revealed a novel decrease in the size of the muscle response to corticospinal tract stimulation during fatigue. This decrease is consistent with a decrease in the excitability of motoneurons and may be a possible contributor to the decline of the discharge rate of single motor units and the development of central fatigue that occur with sustained MVCs. Because the decrease in the CMEP recovers with or without maintained muscle ischemia, it is not likely to be caused by the reflex effects of the increased activity of group III and IV muscle afferents associated with fatiguing contractions. A previous study, in which we also examined CMEPs during a 2 min MVC, did not take into account changes in the size of muscle fiber action potential and so did not reveal the decrease in the CMEP (Gandevia et al., 1996; Taylor et al., 1999).

The magnetic corticospinal tract stimulation used in most experiments here elicits CMEPs of the same latency as those to transmastoid electrical stimulation and is therefore likely to activate axons at a similar cervicomedullary site (Ugawa et al., 1991, 1994; Taylor et al., 2000b, 2002; Petersen et al., 2002). A corticospinal tract stimulus is a useful test of the excitability of the motoneurons because it is not susceptible to changes in presynaptic inhibition that can influence the results from other tests of motoneuronal excitability such as the H-reflex (Nielsen and Petersen, 1994); however, the size of the responses to corticospinal stimulation might also be affected by activity-dependent changes in corticospinal axonal excitability that could change the effectiveness of the stimulus in activating axons, or by changes in the efficacy of connections with motoneurons. In the current study it seems unlikely that the CMEP decrease during the MVC can be accounted for by changes in the corticospinal axonal excitability, because $15 \mathrm{sec}$ after the sustained contraction the responses to corticospinal tract stimulation have recovered. The time course of activity-dependent changes in axonal excitability, as described for human peripheral nerves, is considerably longer (Bergmans, 1970; Vagg et al., 1998; Kiernan et al., 2000). Furthermore, high impulse loads to corticospinal axons produced by pyramidal tract stimulation in monkeys give only minor changes in evoked descending corticospinal volleys (Gandevia et al., 2003).

Similarly, it is unlikely that the depression of the CMEP during a sustained MVC is caused by a reduced efficacy of the corticomotoneuronal synapse. Our previous studies (Gandevia et al.,
Figure 4. Single trials from a typical subject with maintained muscle ischemia. Single responses from brachioradialis to stimulation of the corticospinal tracts (CMEP, left panels) and the brachial plexus ( $M_{\max }$, right panels) from the same subject as in Figure 2 with a period of postcontraction muscle ischemia (dotted box labeled (UFF). Responses are recorded during brief control MVCs (top 3 traces), during the 2 min sustained MVC (next 8 traces), brief recovery MVCs during maintained muscle ischemia (next 4 traces), and during further brief recovery MVCs when the cuff producing ischemia has been released (next 4 traces). The time of corticospinal tract stimulation is indicated on the left, with brachial plexus stimulation $5 \mathrm{sec}$ later. 
1999; Petersen et al., 2003) suggest that the ability of a single descending volley to activate the motoneurons is reduced for up to 2 min after maximal voluntary activation of the motoneurons when tested with the muscle at rest but not when tested during brief MVCs. The postcontraction depression of the CMEP is probably caused by an effect at the corticomotoneuronal synapse rather than the motoneurons themselves, because it does not occur after $30 \mathrm{~Hz}$ maximal stimulation of the peripheral nerve (which would activate motoneurons antidromically and transsynaptically). In the present study, the motoneurons were always tested during MVCs, and although we observed depression of the responses to stimulation of the corticospinal tract during the sustained MVC, there was no postcontraction depression of the response. The difference in the time course of recovery suggests that the intracontraction depression reported in the current study and the postcontraction depression reported in previous studies (Gandevia et al., 1999; Taylor et al., 2000c) are not caused by the same mechanism (Petersen et al., 2003). If synaptic efficacy were reduced during the sustained MVC, it should continue to be reduced during brief MVCs in the recovery period (Gandevia et al., 1999). Decreased synaptic efficacy may only be apparent during relaxation or submaximal contractions (Petersen et al., 2001, 2003).

Therefore, we propose that the decrease in the response to stimulation of the corticospinal tract is caused by decreased excitability of the motoneurons during the 2 min sustained MVC. The data from the present study do not support the hypothesis that group III and IV muscle afferents cause this decrease in excitability (Taylor et al., 2000c). The decrease that we observed in the response of the motoneurons to stimulation of the corticospinal tracts during sustained MVCs recovered despite maintained muscle ischemia. This suggests that group III and IV muscle afferents do not directly inhibit the $\alpha$-motoneuron pool of the elbow flexors in human subjects and that the decrease of the CMEP during the sustained MVC was not caused by the discharge of these afferents. This contrasts with a previous finding that H-reflexes may be diminished with fatigue under ischemic conditions (Garland and McComas, 1990; Garland, 1991). The latter result may be caused by changes in presynaptic inhibition of group Ia afferents by group III and IV muscle afferents (Pettorossi et al., 1999) or changes in the H-reflex afferent volley under these conditions, produced for example by activitydependent changes in axonal excitability (Vagg et al., 1998; for review, see Burke and Gandevia, 1999).

There are at least two other possible contributors to decreased motoneuronal excitability during fatigue. First, changes in other reflex pathways may be relevant. In particular, muscle spindle firing rates decline during fatiguing contractions (Bongiovanni and Hagbarth, 1990; Macefield et al., 1991) and thus reduce reflex facilitation to the contraction. Second, changes in intrinsic motoneuronal properties occur. In animals, when motoneurons are repetitively stimulated by sustained intracellular or extracellular

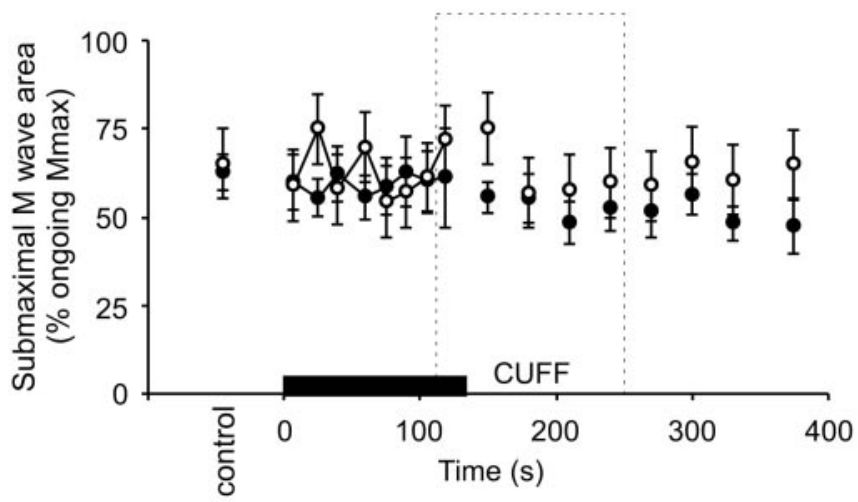

Figure 6. Responses to submaximal brachial plexus stimulation. Mean ( \pm SEM) area of responses (\% ongoing $M_{\max }$ ) from brachioradialis evoked by submaximal stimulation of the brachial plexus ( $n=8$ subjects). Data are shown from experiments both without (filled circles) and with (open circles) maintained muscle ischemia for $2 \mathrm{~min}$ (indicated by dashed box labeled CUFF) after the 2 min MVC (indicated by black bar). Because the ratio remains constant throughout both of the experiments, the data suggest that sarcolemmal changes attributable to fatigue or maintained muscle ischemia affected all muscle fiber types similarly.

currents, many of the cells either reduce their discharge or stop firing (Kernell and Monster, 1982a,b; Spielmann et al., 1993; Sawczuk et al., 1997). In humans, repetitive antidromic activation of motoneurons also reduces motoneuron excitability assessed with F-waves (Butler and Thomas, 2003). Effectively, after repetitive activation, a greater input is required to produce the same output from the motoneurons. Reduced CMEP responses are also consistent with the motoneurons having an increased duration of afterhyperpolarization associated with their lower 
firing rates (Matthews, 1999). Given that the CMEP decreases during a sustained MVC, our data are also consistent with the motoneurons adapting and becoming less excitable during sustained drive, such that they become harder to activate through a descending corticospinal volley.

In addition, our findings reveal a potential paradox. During maintained muscle ischemia after a fatiguing contraction, the firing rates of motoneurons and maximal voluntary activation of muscles do not recover (Bigland-Ritchie et al., 1986; Woods et al., 1987; Gandevia et al., 1996), yet motoneuron excitability as tested here (with a CMEP) does recover. These findings could be reconciled if there is recovery during the rest periods of the depression of motoneuron excitability produced by prolonged repetitive activation, while corticospinal drive and muscle spindle drive stay reduced during maximal efforts during maintained ischemia as a result of maintained firing of group III and IV muscle afferents. Indeed, there is evidence that descending command is suboptimal under these ischemic conditions, and additional output is available from the motor cortex, because motor cortical stimulation can transiently increase force (Gandevia et al., 1996). The net facilitation provided by muscle afferents is probably also reduced (Bongiovanni and Hagbarth, 1990; Gandevia et al., 1990; Macefield et al., 1993); however, other explanations are possible, and the paradox highlights the difficulty in measurement of motoneuronal excitability in studies in human subjects.

\section{References}

Allen GM, Gandevia SC, McKenzie DK (1995) Reliability of measurements of muscle strength and voluntary activation using twitch interpolation. Muscle Nerve 18:593-600.

Bergmans J (1970) The physiology of single human nerve fibres. Vander, Belgium: University of Louvain.

Bigland-Ritchie B, Johansson R, Lippold OCJ, Woods JJ (1983a) Contractile speed and EMG changes during fatigue of sustained maximal voluntary contractions. J Neurophysiol 50:313-324.

Bigland-Ritchie B, Johansson R, Lippold OCJ, Smith S, Woods JJ (1983b) Changes in motoneurone firing rates during sustained maximal voluntary contractions. J Physiol (Lond) 340:335-346.

Bigland-Ritchie B, Dawson NJ, Johansson R, Lippold OCJ (1986) Reflex origin for the slowing of motoneurone firing rates in fatigue of human voluntary contractions. J Physiol (Lond) 379:451-459.

Bigland-Ritchie B, Rice CL, Garland SJ, Walsh ML (1995) Task-dependent factors in fatigue of human voluntary contractions. Adv Exp Med Biol 384:361-380.

Bongiovanni LG, Hagbarth KE (1990) Tonic vibration reflexes elicited during fatigue from maximal voluntary contractions in man. J Physiol (Lond) 423:1-14.

Burke D, Gandevia SC (1999) Properties of human peripheral nerves: implications for studies of human motor control. In: Peripheral and spinal mechanisms in the neural control of movement (Binder MD, ed), pp 427-435. Amsterdam: Elsevier.

Butler JE, Thomas CK (2003) Effects of sustained electrical stimulation on the excitability of motoneurons innervating paralyzed and control muscles. J Appl Physiol 94:567-575.

Butler JE, Taylor JL, Gandevia SC (1999) Responses to stimulation of corticospinal axons are reduced during sustained voluntary contractions in humans. Soc Neurosci Abstr 25:50-54.

Cupido CM, Galea V, McComas AJ (1996) Potentiation and depression of the $\mathrm{m}$ wave in human biceps brachii. J Physiol (Lond) 491:541-550.

Enoka RM, Trayanova N, Laouris Y, Bevan L, Reinking RM, Stuart DG (1992) Fatigue-related changes in motor unit action potentials of adult cats. Muscle Nerve 15:138-150.

Gandevia SC, Macefield G, Burke D, McKenzie DK (1990) Voluntary activation of human motor axons in the absence of muscle afferent feedback. The control of the deafferented hand. Brain 113:1563-1581.

Gandevia SC, Allen GM, Butler JE, Taylor JL (1996) Supraspinal factors in human muscle fatigue: evidence for suboptimal output from the motor cortex. J Physiol (Lond) 490:529-536.

Gandevia SC, Petersen N, Butler JE, Taylor JL (1999) Impaired response of human motoneurones to corticospinal stimulation after voluntary exercise. J Physiol (Lond) 521:749-759.

Gandevia SC, Shimazu H, Maier MA, Kirkwood PA, Lemon RN (2003) Effects of impulse trains on corticospinal conduction in monkeys. Proc Aust Neurosci Soc 14:06-07.

Garland SJ (1991) Role of small diameter afferents in reflex inhibition during human muscle fatigue. J Physiol (Lond) 435:547-558.

Garland SJ, Gossen ER (2002) The muscular wisdom hypothesis in human muscle fatigue. Exerc Sport Sci Rev 30:45-49.

Garland SJ, McComas AJ (1990) Reflex inhibition of human soleus muscle during fatigue. J Physiol (Lond) 429:17-27.

Grimby L, Hannerz J, Hedman B (1981) The fatigue and voluntary discharge properties of single motor units in man. J Physiol (Lond) 316:545-554.

Hayward L, Breitbach D, Rymer WZ (1988) Increased inhibitory effects on close synergists during muscle fatigue in the decerebrate cat. Brain Res 440:199-203.

Hayward L, Wesselmann U, Rymer WZ (1991) Effects of muscle fatigue on mechanically sensitive afferents of slow conduction velocity in the cat triceps surae. J Neurophysiol 65:360-370.

Kaufman MP, Rybicki KJ, Waldrop TG, Ordway GA (1984) Effect of ischaemia on responses of group III and IV afferents to contraction. J Appl Physiol 57:644-650.

Kernell D, Monster AW (1982a) Time course and properties of late adaptation in spinal motoneurones of the cat. Exp Brain Res 46:191-196.

Kernell D, Monster AW (1982b) Motoneurone properties and motor fatigue: an intracellular study of gastrocnemius motoneurones of the cat. Exp Brain Res 46:197-204.

Kiernan MC, Burke D, Andersen KV, Bostock H (2000) Multiple measures of axonal excitability: a new approach in clinical testing. Muscle Nerve 23:399-409.

Kniffki KD, Schomburg ED, Steffens H (1979) Synaptic responses of lumbar alpha-motoneurones to chemical algesic stimulation of skeletal muscle in spinal cats. Brain Res 160:549-552.

Kniffki KD, Schomburg ED, Steffens H (1981a) Synaptic effects from chemically activated fine muscle afferents upon alpha-motoneurones in decerebrate and spinal cats. Brain Res 206:361-370.

Kniffki KD, Schomburg ED, Steffens H (1981b) Convergence in segmental reflex pathways from fine muscle afferents and cutaneous or group II muscle afferents to alpha-motoneurones. Brain Res 218:342-346.

Macefield G, Hagbarth KE, Gorman R, Gandevia SC, Burke D (1991) Decline in spindle support to alpha-motoneurones during sustained voluntary contractions. J Physiol (Lond) 440:497-512.

Macefield VG, Gandevia SC, Bigland-Ritchie B, Gorman RB, Burke D (1993) The firing rates of human motoneurones voluntarily activated in the absence of muscle afferent feedback. J Physiol (Lond) 471:429-443.

Macefield VG, Fuglevand AJ, Howell JN, Bigland-Ritchie B (2000) Discharge behaviour of single motor units during maximal voluntary contractions of a human toe extensor. J Physiol (Lond) 528:227-234.

Marsden CD, Meadows JC, Merton PA (1969) Muscular wisdom. J Physiol (Lond) 200:15P.

Marsden CD, Meadows JC, Merton PA (1983) "Muscular wisdom" that minimises fatigue during prolonged effort in man: peak rates of motoneurone discharge and slowing of discharge during fatigue. Adv Neurol 39:169-211.

Matthews PBC (1999) The effect of firing on the excitability of a model motoneurone and its implications for cortical stimulation. J Physiol (Lond) 518:867-882.

Nielsen J, Petersen N (1994) Is presynaptic inhibition distributed to corticospinal fibres in man? J Physiol (Lond) 477:47-58.

Petersen NT, Taylor JL, Butler JE, Gandevia SC (2001) Reduced corticospinal transmission to motoneurones after strong voluntary contraction persists during a weak contraction. Proc Aust Neurosci Soc 12:339P.

Petersen NT, Taylor JL, Gandevia SC (2002) The effect of electrical stimulation of the corticospinal tract on motor units of human biceps brachii. J Physiol (Lond) 544:277-284.

Petersen N, Taylor JL, Butler JE, Gandevia SC (2003) Depression of activity in the corticospinal pathway during human motor behavior after strong voluntary contractions. J Neurosci 23:7974-7980.

Pettorossi VE, Della Torre G, Bortolami R, Brunetti O (1999) The role of 
capsaicin-sensitive muscle afferents in fatigue-induced modulation of the monosynaptic reflex in the rat. J Physiol (Lond) 515:599-607.

Sacco P, Newberry R, Mcfadden L, Brown T, McComas AJ (1997) Depression of human electromyographic activity by fatigue of a synergistic muscle. Muscle Nerve 20:710-717.

Sawczuk A, Powers RK, Binder MD (1997) Contributions of outward currents to spike frequency adaptation in hypoglossal motoneurones of the rat. J Neurophysiol 78:2246-2253.

Spielmann JM, Laouris Y, Nordstrom MA, Robinson GA, Reinking RM, Stuart DG (1993) Adaptation of cat motoneurons to sustained and intermittent extracellular activation. J Physiol (Lond) 464:75-120.

Taylor JL, Butler JE, Gandevia SC (1999) Altered responses of human elbow flexors to peripheral-nerve and cortical stimulation during a sustained maximal voluntary contraction. Exp Brain Res 127:108-115.

Taylor JL, Allen GM, Butler JE, Gandevia SC (2000a) Supraspinal fatigue during intermittent maximal voluntary contractions of the human elbow flexors. J Appl Physiol 89:305-313.

Taylor JL, Butler JE, Gandevia SC (2000b) Magnetic stimulation of the descending tracts in human subjects. Proc Aust Neurosci Soc 11:48.

Taylor JL, Petersen N, Butler JE, Gandevia SC (2000c) Ischaemia after exer- cise does not reduce responses of human motoneurones to cortical or corticospinal tract stimulation. J Physiol (Lond) 525:793-801.

Taylor JL, Petersen NT, Butler JE, Gandevia SC (2002) Interaction of transcranial magnetic stimulation and electrical transmastoid stimulation in human subjects. J Physiol (Lond) 541:949-958.

Ugawa Y, Rothwell JC, Day BL, Thompson PD, Marsden CD (1991) Percutaneous electrical stimulation of corticospinal pathways at the level of the pyramidal decussation in humans. Ann Neurol 29:418-427.

Ugawa Y, Uesaka Y, Terao Y, Hanajima R, Kanazawa I (1994) Magnetic stimulation of corticospinal pathways at the foramen magnum level in humans. Ann Neurol 36:618-624.

Vagg R, Mogyoros I, Kiernan MC, Burke D (1998) Activity-dependent hyperpolarization of human motor axons produced by natural activity. J Physiol (Lond) 507:919-925.

Walton DM, Kuchinad RA, Ivanova TD, Garland SJ (2002) Reflex inhibition during muscle fatigue in endurance-trained and sedentary individuals. Eur J Appl Physiol 87:462-468.

Woods JJ, Furbush F, Bigland-Ritchie B (1987) Evidence for a fatigueinduced reflex inhibition of motoneuron firing rates. J Neurophysiol 58: 125-137. 